\title{
Predictive factors associated with the clinical outcome of intertrochanteric hip fracture in high-risk elderly patients treated with total hip arthroplasty versus percutaneous external fixation
}

\author{
Jun Zhang ${ }^{1 \# \wedge}$, Jie Zhu ${ }^{2 \#}$, Zaiyang Liu ${ }^{1}$, Yumei Zhang ${ }^{1}$, Yunfei Jin ${ }^{3}$, Min Wang ${ }^{1}$, Xia Zhang ${ }^{1}, K^{2}$ aiqi He ${ }^{1}$, \\ Yuan Zhang ${ }^{1 \wedge}$
}

${ }^{1}$ Joint Disease \& Sport Medicine Center, Department of Orthopedics, Xinqiao Hospital, Army Medical University, Chongqing, China; ${ }^{2}$ Department of Neurology, Daping Hospital, Army Medical University, Chongqing, China; ${ }^{3}$ Peking University Clinical Research Institute, Peking University First Hospital, Beijing, China

Contributions: (I) Conception and design: Y Zhang, J Zhu; (II) Administrative support: M Wang, X Zhang; (III) Provision of study materials or patients: Y Zhang, M Wang, X Zhang; (IV) Collection and assembly of data: J Zhang, Z Liu, K He; (V) Data analysis and interpretation: Y Jin, J Zhang, YM Zhang; (VI) Manuscript writing: All authors; (VII) Final approval of manuscript: All authors.

"These authors contributed equally to this work.

Correspondence to: Yuan Zhang, MD, PhD. 183 Xinqiao Street, Shapingba District, Chongqing 400038, China. Email: zhangyuan@tmmu.edu.cn; joint_chueng@hotmail.com.

Background: Little is known regarding the survival and functional recovery of elderly intertrochanteric hip fracture (IHF) patients after total hip arthroplasty (THA) versus percutaneous external fixation (PEF). This study aims to analyze the prognostic factors of THA and PEF in elderly IHF patients.

Methods: A total of 155 consecutive elderly patients (mean age of 80 years) diagnosed with IHF were retrospectively reviewed from our database between January 1, 2010, and December 31, 2018. The preoperative, intraoperative and postoperative covariates were analyzed by two independent surgical cohorts: THA and PEF. The main outcomes included the hip function score, all-cause mortality within 1 year after surgery, and overall survival. Covariables and their influence on independent outcomes were analyzed using multivariate regression models.

Results: The median follow-up period was 5.1 years, and 6 patients were lost to follow-up. At the endpoint, 70 of 85 patients treated with THA and 37 of 70 patients treated with PEF survived, exhibiting mean Harris hip scores of 84.4 and 69.0, respectively. The Kaplan-Meier curves and log-rank tests showed no significant difference in overall survival. After adjusting for the covariates, the surgical mode was a unique prognostic factor affecting hip function recovery, and two prognostic factors (leukocyte count and D-dimer) were correlated with 1-year all-cause mortality. Age at admission, fracture classification, D-dimer level and surgical mode were identified as prognostic factors affecting overall survival. After adjusting for the former three covariates, THA reduced the risk of death by $67.20 \%$ compared with PEF (HR 0.328, 95\% CI, 0.121-0.890).

Conclusions: Despite the nonsignificant difference in 1-year all-cause mortality, THA demonstrated superior midterm survival and hip function recovery in elderly IHF patients compared with PEF. Predictive factors, including age at admission, fracture classification, D-dimer level and surgical mode, are associated with the overall survival of IHF in high-risk elderly patients.

Keywords: Intertrochanteric hip fractures (IHFs); elderly; arthroplasty; external fixation; survival; functional recovery; early mortality

Submitted Dec 22, 2020. Accepted for publication Mar 17, 2021.

doi: $10.21037 / \mathrm{atm}-20-8037$

View this article at: http://dx.doi.org/10.21037/atm-20-8037

^ ORCID: Yuan Zhang, 0000-0003-3341-2995; Jun Zhang, 0000-0002-1122-3811. 


\section{Introduction}

The incidence of hip fracture and associated mortality, medical expenses and social burden in elderly patients has increased considerably in the last decade. An epidemiological investigation showed that 1.6 million cases occur annually worldwide, with a predicted occurrence of 6.3 million by $2050(1-3)$. The prognosis is usually poor due to a mortality rate of $10 \%$ at 1 month and $30 \%$ at 1 year $(4,5)$. A total of $38-50 \%$ of hip fractures occur in the intertrochanteric region (6). Although there is much similarity in the pathology of intertrochanteric hip fracture (IHF) and femoral neck fracture, the different treatment modes could be used (7). There is a strong consensus regarding the treatment of femoral neck fracture by hip arthroplasty, which has been proven to be superior in terms of quality of life and long-term survival $(8,9)$.

However, there are notable controversies regarding the roles of repair strategies, such as fracture fixation, other than reconstructive approaches, such as arthroplasty, in patients diagnosed with IHF (10-13). Classical internal fixation (dynamic hip screw, proximal femoral nail and locking plate) has been recommended as another choice for elderly IHF patients due to the high failure rate (range, $4.7-16.5 \%)$ and poor general condition of this population, preventing anesthesia for an invasive intervention (14-17). Hip arthroplasty, either hemiarthroplasty (HA) or total hip arthroplasty (THA), is indicated in unstable IHF, patients with preexisting disorders (such as osteoarthritis), patients suffering from bedridden complications (bedsore, respiratory and urinary infection, etc.) and patient asking for immediate weight-bearing activities (18). However, a high frequency of complications, such as dislocation, implant loosening and abductor insufficiency, is the subject of many complaints (19-21). Arthroplasty is mostly recommended in elderly patients with unstable osteoporotic fractures or as a salvage procedure in cases of fixation failure $(22,23)$. Another choice is closed reduction and percutaneous external fixation (PEF), which can be applied under local anesthesia with sedation and has been reported to be appropriate for high-risk patients (24-27). Many studies have documented that PEF could be cost-effective in delaying surgery and reducing the operative duration, blood loss and surgical trauma $(25,26,28)$. However, little is known regarding its long-term outcome in elderly patients.

Many studies have compared the clinical outcomes between internal fixation and hip arthroplasty, and the results remain controversial $(7,9-11,13,15,20,22)$. We undertook a retrospective cohort study to compare the therapeutic effect of two mainstay treatment modes, PEF and THA, in elderly patients between July 1, 2010, and December 31, 2018. Our study aimed to answer two questions: first, is there any clinical difference in terms of functional independence and survival rate; and second, which are the prognostic factors affecting the main outcomes, including hip function recovery, all-cause mortality within one year after surgery, and endpoint survival? We present the following article in accordance with the STROBE reporting checklist (available at http:// dx.doi.org/10.21037/atm-20-8037).

\section{Methods}

\section{Data sources and study design}

The cohort comprised all consecutive patients diagnosed with IHF who underwent PEF or THA at our institution from January 1, 2010, through December 31, 2018. All of the data were retrieved by a picture archiving and communication system (PACS) station (version 10.0) using ICD-11-CM diagnosis codes for IHF with approval from the institutional review board. The search was further defined by the following inclusion criteria: (I) age over 75 years old at admission; (II) unilateral and closed IHF within 3 weeks of injury; and (III) IHF confirmed according to the Evan classification system (29). The exclusion criteria were as follows: (I) elderly IHF patients with coexisting hip disorders, such as osteoarthritis and femoral head avascular necrosis; (II) preinjury nonambulatory status; (III) contralateral hip fracture during follow-up; and (IV) treatment with HA. The study was conducted in accordance with the Declaration of Helsinki (as revised in 2013). The study was approved by the institutional committee of Xinqiao Hospital (No.: 2019027), and individual consent for this retrospective analysis was waived.

Two groups of patients treated by surgical modes of PEF and THA were set as the comparative cohorts. The choice of the surgical mode was made by the patient and his/her family member after a risk-benefit assessment and therapeutic advice were proposed by a multidisciplinary team. All surgical procedures were performed by three senior surgeons (X.Z., M.W., and Y.Z.). The PEF was accomplished by fracture reduction by axial traction and internal rotation of the injured limb in a tractable system under fluoroscopy monitoring, followed by fixing the fracture with two $4.5 \mathrm{~mm}$ cancellous Schanz screw 
transfracture lines percutaneously and two $4.5 \mathrm{~mm}$ cortical Schanz screws on the femoral shaft. THA was performed via minimally invasive Moore's approach in a lateral decubitus position, with sequential procedures consisting of repositioning and temporary fixation of the fragments of the trochanter, preparation of the acetabulum and femoral canal, implantation of cementless and fully coated porous components, and repair of the short external rotators.

\section{Covariates}

Baseline characteristics and preoperative assessment Patient age, sex, operational side, body mass index (BMI), cause of injury, fracture classification and anesthesia type were collected from PACS files. Validated algorithms identified patients with four types of preexisting disorders, including cardiovascular disease (coronary and cerebral vascular disease, hypertension, etc.), respiratory disease (chronic obstructive pulmonary disease, hypostatic pneumonia, etc.), metabolic disease (diabetes, hyperthyroidism, etc.), and neurological disease (Parkinson's disease, Alzheimer's disease, etc.). The preoperative risk ratings, including bone quality, American Society of Anesthesia (ASA) score, nutritional risk screening (NRS2002), Caprini score of venous thromboembolism, bedsore risk rating (Braden score), self-care and independent ability (ADL-Barthel score), and mental status (Nu-DESC score), were retrieved (30-33).

\section{Intraoperative and postoperative data}

The intraoperative descriptive variables included inhospital length of stay, corrected medical expenses (subtracting the implant expense due to the significant difference in expense of the external fixator and arthroplasty prosthesis), operative duration, blood loss, and transfusion volume and rate. The one-day postoperative descriptive variables included leukocyte count, neutrophil percentage, albumin and D-dimer levels, blood oxygen pressure and oxygen saturation rate. Adverse events were defined by complications related to hip surgery within three months postoperatively and the causes of unexpected death within the first year postoperatively.

\section{Follow-up variables}

All patients were followed up by clinical and radiological evaluation annually, and the endpoint of the follow-up period was set as the start of the statistical analysis of this study (December 31, 2019). The variables employed for evaluating functional outcomes and quality of life were the Harris Hip Score (HHS) and Short Form 12 Health Survey (SF-12) score. Radiological analysis was employed to determine functional bone healing and the occurrence of implant failure, including bone nonunion, mechanical loosening, loss of fracture reduction, implant loosening, cutting-out, and subsidence.

\section{Outcome metrics}

The primary outcome was overall survival at the final follow-up. The secondary outcomes included hip function recovery indicated by the HHS and all-cause mortality within 1 year after surgery.

\section{Statistical analysis}

Statistical analyses were performed using SAS Version 9.4 (SAS Institute, Cary, NC). Continuous variables are described as the mean and standard deviation (SD), and nonnormally distributed data are described as the median and interquartile range. Twosided tests were performed for all statistical analyses. CochranMantel-Haenszel $\chi^{2}$ tests or Fisher's exact tests were used to compare categorical data. Continuous data were analyzed by Student's $t$-test or Wilcoxon rank test. P values of 0.05 were considered to indicate statistical significance.

Kaplan-Meier survival curves and a log-rank test were used to compare survival between the two groups. The specific prognostic factors with clinical outcomes were determined by three different regression models: (I) a multiple linear regression model for associated prognostic factors of hip functional recovery (HHS); (II) a logistic regression model for associated prognostic factors of allcause mortality within one year after surgery; and (III) a multivariate Cox proportional hazards model for associated prognostic factors of general survival.

\section{Results}

\section{Data sources and patient characteristics}

A total of 252 patients were identified in the database according to our inclusion criteria, and 78 cases were excluded as follows: 32 cases of combined femoral head necrosis and hip osteoarthritis, 17 cases of contralateral hip fracture after hip surgery, and 29 cases of nonambulatory preinjury status. The complete medical documents of the remaining 155 patients were reviewed from January 1, 2010 through December 31, 2018. The median follow-up period 
Table 1 Baseline characteristics and preoperative risk assessment of the patients in this study

\begin{tabular}{|c|c|c|c|}
\hline Indicator & THA $(n=85)$ & $\operatorname{PEF}(n=70)$ & $\mathrm{P}$ \\
\hline Age at admission [mean (SD)], year & $80.0(7.1)$ & $83.6(7.1)$ & 0.963 \\
\hline Operational side (left/right), case & $41 / 44$ & $38 / 32$ & 0.455 \\
\hline Body mass index, [mean (SD)] & $20.9(3.2)$ & $21.6(3.8)$ & 0.460 \\
\hline Cause of injury $(\mathrm{I} / \mathrm{II} / \mathrm{III})^{*}$, case & $82 / 0 / 3$ & $63 / 4 / 3$ & 0.098 \\
\hline Fracture classification $(\mathrm{I} / \mathrm{II} / \mathrm{III} / \mathrm{IV} / \mathrm{V} / \mathrm{R})^{\star}$, case & $2 / 13 / 11 / 18 / 40 / 1$ & $0 / 8 / 13 / 18 / 27 / 4$ & 0.318 \\
\hline Time from injury to surgery*, day & $3.5 / 7 / 10.5$ & $3 / 4 / 7$ & 0.001 \\
\hline Preoperative comorbidities, case & $49 / 58 / 29 / 17$ & $43 / 52 / 26 / 28$ & 0.235 \\
\hline Pain score (NRS), mean (SD) & $2.0(1.7)$ & $1.6(1.4)$ & 0.215 \\
\hline Nutrition risk (NRS2002), mean (SD) & $2.7(0.9)$ & $2.6(0.8)$ & 0.498 \\
\hline VTE risk (Carprini score) ${ }^{\star}$, mean (SD) & $14 / 16 / 24 / 31$ & $13 / 10 / 12 / 35$ & 0.364 \\
\hline Bedsore risk (Braden score), mean (SD) & $14.1(1.7)$ & $14.4(2.2)$ & 0.407 \\
\hline Self-care ability (ADL-Barthel) ${ }^{\star}$, mean (SD) & $25.6(3.2)$ & $26.7(9.7)$ & 0.769 \\
\hline Mental status (Nu-DESC) ${ }^{*}$, mean (SD) & $1.7(0.4)$ & $1.9(0.6)$ & 0.127 \\
\hline
\end{tabular}

*, bone quality: I, normal (T>-1.0); II, osteopenia $(-2.5<\mathrm{T}<-1.0)$; III, osteoporosis $(\mathrm{T}<-2.5)$; IV, severe osteoporosis $(\mathrm{T}<-2.5$, combined with osteoporotic fracture). Cause of injury: I, fall while walking; II, fall from height; III, road traffic accident. Fracture classification: I/II/III/IV/V/R of EVANS' system. Time from injury to surgery: lower quartile/median/upper quartile. Preoperative comorbidities: cardio-vascular/respiratory/ metabolic/neurological. ADL-Barthel score: I, normal [100]; II, mild [61-99]; III, moderate [41-60]; IV, severe [<40]. VTE risk score: I, low risk; II, moderate risk; III, high risk; IV, extremely high risk. SD, standard deviation; THA, total hip arthroplasty; PEF, percutaneous external fixation; ASA, American Society of Anesthesiologists; NRS, Numerical Rating Scale; NRS2002, nutrition risk screening of 2002; VTE, venous thrombus embolism; ADL, activities of daily living; Nu-DESC, Nursing Delirium Screening Scale.

was 5.1 years (range, 1.5-9.5 years).

Continuous variables presented, including age at admission and BMI, were similar in the two groups. There was no significant difference in categorical variables, including sex, operational side, bone quality, cause of injury, and fracture classification. The quantitative analysis of surgical-related risks, including pain, nutrition, venous thromboembolism, bedsore, self-care ability and mental status risks, also demonstrated no significant difference. We did notice a significant difference in three variables, including the ASA score, anesthesia type and time from injury to surgery (TFIS) (Table 1).

\section{Intraoperative and postoperative variables}

In terms of intraoperative variables, the operative duration (from incision to closure) and estimated blood loss were significantly lower in PEF than in THA $(\mathrm{P}<0.05)$. Although the general transfusion rate was much lower in PEF, the difference in transfusion volume in the two groups remained nonsignificant, and it was calculated from the total blood volume given to the number of patients who received transfusions (13 in PEF versus 60 in THA). The medical expenses and length of in-hospital stay were similar in the two groups $(\mathrm{P}=0.296)$. Blood tests on postoperative day 1 showed significantly higher leukocyte counts and $\mathrm{D}$-dimer levels in THA patients than in PEF patients $(\mathrm{P}<0.05)$, while other hematological variables, including the neutrophil percentage, albumin level, oxygen saturation and blood oxygen pressure, were identical $(\mathrm{P}>0.05)$. The total postoperative complication rate was $22.3 \%$ in THA versus $26.6 \%$ in $\mathrm{PEF}(\mathrm{P}=0.552)$. In particular, postoperative dislocation was exclusively found in THA, with a significant 
difference $(\mathrm{P}=0.017$, Table 2).

\section{Association of covariates and bip function recovery}

At the endpoint of the study, the HHS was 84.4 and 69.0 in the THA and PEF groups, respectively $(\mathrm{P}<0.001)$, and the percentage of patients whose hip function reached a grade of excellent and fine was significantly higher in the THA group $(\mathrm{P}<0.01)$. In terms of overall quality of life, the SF-12 physical component score increased by 16.2 versus 14.1 points in THA and $\mathrm{PEF}$, respectively $(\mathrm{P}<0.001)$, and no significance was observed in the mental component. We then determined prognostic factors affecting the HHS using a specific regression model. We found that the surgical mode had a significant influence on the final hip function score using a linear regression model $(\mathrm{P}=0.002)$. Moreover, we assumed that the HHS at the final follow-up was likely to be associated with relevant prognostic factors. The results showed that the surgical mode was a unique prognostic factor affecting the final hip function score after adjusting for covariates using a multiple regression model $(\mathrm{P}=0.04$, Table 3$)$.

\section{Association of covariates and all-cause mortality within 1 year after surgery}

Our primary investigation demonstrated that the rate of all-cause mortality within 1 year after THA was $5.9 \%$ $(5 / 85)$, and the four most common causes were respiratory failure $(50 \%)$, cardiac failure (12.5\%), pulmonary embolism $(12.5 \%)$ and sepsis $(12.5 \%)$. In contrast, the rate of all-cause mortality within 1 year after PEF was 11.4\% (8/70), and the three most common causes were respiratory failure $(40 \%)$, cancer (20\%), and pulmonary embolism (20\%). There was no significant difference in the 1-year mortality rate $(\mathrm{P}=0.261)$. In addition, we preliminarily identified that the surgical mode had no significant impact on this mortality rate through logistic regression analysis $(\mathrm{P}=0.228)$.

However, when we introduced associated factors, including the ASA score, TFIS, leukocyte count, hemoglobin, albumin and D-dimer levels at 1 day after surgery, and coexisting comorbidities into the logistic regression model, the results showed that the leukocyte count and D-dimer level were prognostic factors affecting all-cause mortality within 1 year after surgery $(\mathrm{P}<0.001$, Table 4$)$.

\section{Association of covariates and overall survival}

Seventy of $85(82.3 \%)$ patients in the THA group survived, with 15 cases of death, while 37 of $70(52.9 \%)$ patients in the PEF group survived, with 27 cases of death and 6 patients lost to follow-up at the median period of 5.1 years. A log-rank test without adjusting for covariates was used to evaluate the overall survival in the two groups. There was no significant difference in overall survival between the two groups $(\mathrm{P}=0.0669$, Figure 1). Patients treated with $\mathrm{PEF}$ had a median overall survival of 7.1 years [95\% confidence interval (CI), 5.4-10.2], while patients treated with THA had a median survival time of more than 9.5 years (95\% CI, 7.9-13.2).

Four variables, including age at admission, fracture classification, D-dimer level and surgical mode, had a significant prognostic influence on overall survival (Table 5). After adjusting for the former three variables, the results suggested a significant risk ratio for THA versus PEF (HR $0.328,95 \%$ CI, 0.121-0.890), revealing that THA has a mortality $67.2 \%$ less than that of PEF.

\section{Discussion}

The treatment of elderly IHF patients is challenging in terms of both decision-making and perioperative management $(34,35)$. There are still massive debates on the topics of surgical mode, implant selection, intervention time, and perioperative management $(36,37)$. This study highlights the value of the surgical mode in this high-risk population.

There have been very few studies on the mid- to longterm clinical outcomes in high-risk patients treated with THA versus PEF. Polat et al. observed the short-term outcome of PEF versus HA in 42 elderly unstable IHF patients. They found PEF to be an advantageous choice compared with HA in terms of a superior surgical time, hospital stay, and intensive care unit stay. The one-year mortality rates were $55.0 \%$ and $40.9 \%$ in the PEF and HA groups, respectively. Both the survival rate and functional results were not different at the last follow-up visit (38). In our study, we enrolled all consecutive patients diagnosed with IHF and surgically treated with THA and PEF at our center from 2010 to 2018, illustrated the clinical outcome of the two surgical modes and further unveiled the definite prognostic factors related to the outcome.

The first significant finding that we identified is a difference in the midterm outcome of elderly IHF patients treated with THA and PEF. We proved that the overall survival rate at the 5.1-year follow-up in the THA group was significantly higher than that in the PEF group, with an estimated difference of $30.5 \%$ in our retrospective series. 
Table 2 Intra- and post-operative variables, follow-up records, and complication of this study

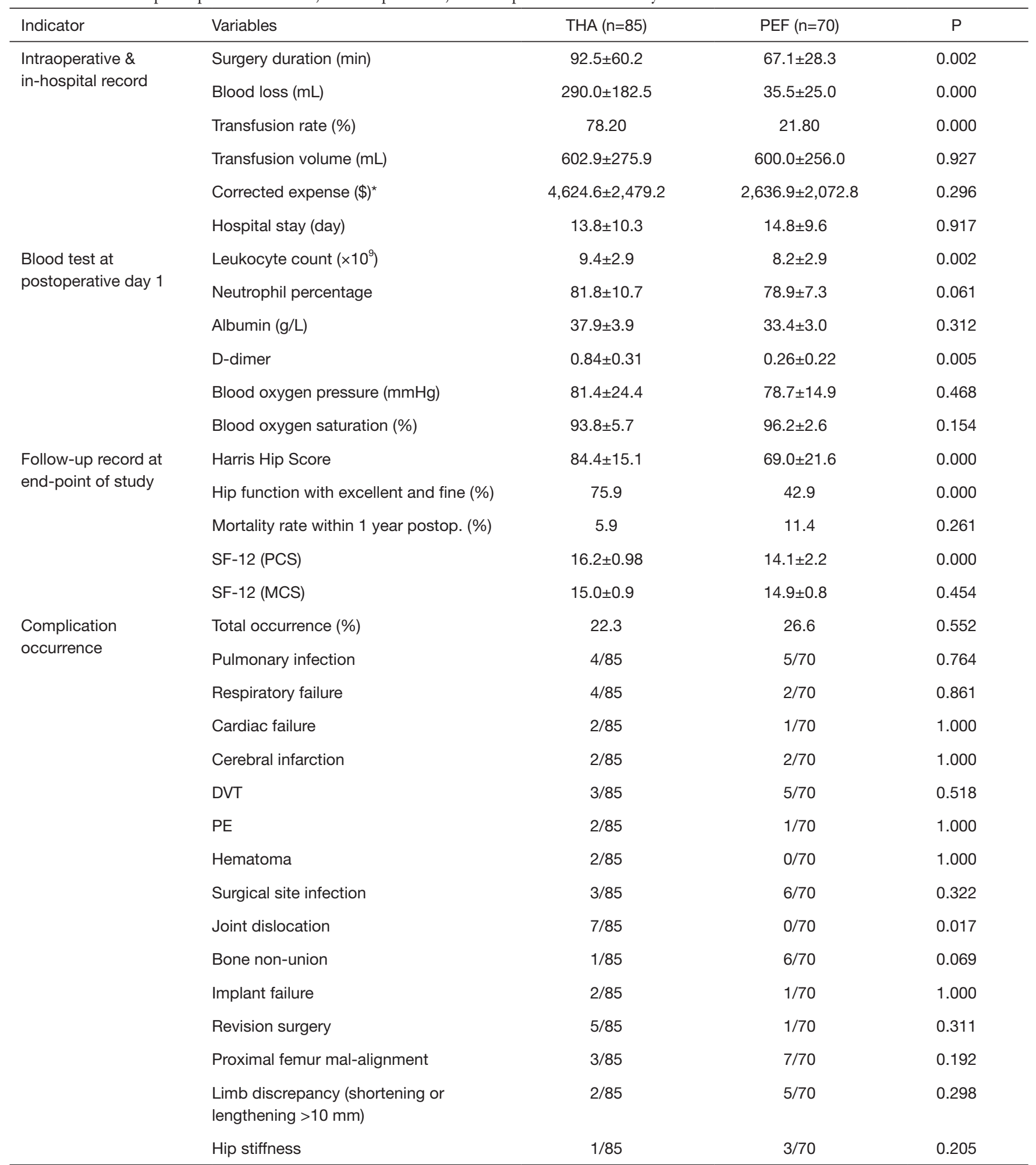

*, medical expense after subtracting the implant expense from total expense. THA, total hip arthroplasty; PEF, percutaneous external fixation; SF-12, 12-item short form health survey; PCS, physical component score; MCS, mental component score; DVT, deep venous thrombosis; PE, pulmonary embolism. 
Table 3 Association of prognostic factors with Harris Hip Score

\begin{tabular}{lcccc}
\hline Variates & III type SS & MS & F value & P \\
\hline ASA score & 98.497 & 98.497 & 0.41 & 0.521 \\
Fracture classification & 656.074 & 131.215 & 0.55 & 0.736 \\
Anesthesia type & 189.911 & 189.911 & 0.80 & 0.374 \\
Respiratory disorder & 0.961 & 0.961 & 0.00 & 0.949 \\
Metabolic disorder & 44.365 & 44.365 & 0.19 & 0.667 \\
Cardio-vascular disorder & 0.0482 & 0.048 & 0.00 & 0.989 \\
Neurological diseases & 128.154 & 128.154 & 0.54 & 0.465 \\
Surgical mode & $1,016.164$ & $1,016.164$ & 4.28 & 0.041 \\
\hline
\end{tabular}

ASA, American Society of Anesthesiologists; SS, sums of squares; MS, mean squared.

Table 4 Association of prognostic factors and mortality from any reason within 1 year after surgery

\begin{tabular}{|c|c|c|c|c|}
\hline Variates & $B$ value & SE & Wald & $\mathrm{P}$ \\
\hline ASA score & -1.997 & 1.156 & 2.984 & 0.084 \\
\hline TFIS & -0.264 & 0.581 & 0.207 & 0.649 \\
\hline Leukocyte count & -1.423 & 0.517 & 7.588 & 0.006 \\
\hline Hemoglobin & -0.199 & 0.194 & 1.057 & 0.304 \\
\hline Albumin & -0.231 & 0.330 & 0.488 & 0.485 \\
\hline Metabolic disorder & 2.565 & 1.972 & 1.692 & 0.193 \\
\hline Cardio-vascular disorder & 0.047 & 1.405 & 0.001 & 0.974 \\
\hline Surgical mode & -4.1710 & 2.6647 & 2.4501 & 0.1175 \\
\hline
\end{tabular}

SE, standard error; ASA, American Society of Anesthesiologists; TFIS, time from injury to surgery.

We further estimated that THA would have a 5.1-year mortality rate after surgery $67.2 \%$ less than that of PEF using a Cox proportional hazards model.

This finding is typically contradictory to the traditional viewpoint of the role of PEF in elderly IHF patients. Although many studies have recommended PEF as an optimal choice for these patients because of economic and comorbidity-related considerations $(38,39)$, their conclusions were inclusive because the findings were obtained in the acute phase (perioperative period) or after short-term observation. To our knowledge, we are the first to illustrate in this retrospective study that PEF in elderly IHF patients has inferior midterm outcomes compared with THA.

The second significant finding is that we screened extensive covariates related to the surgical mode and identified definite prognostic factors affecting overall survival and hip functional recovery at the last follow-up as well as all-cause mortality within 1 year after surgery. The factors were included in our regression analysis based on the study objectives, clinical judgment and literature review. These factors included the following: baseline patient factors, preoperative risk assessment results and comorbidities, intraoperative and postoperative variables, and incidence of complications. To our knowledge, this 


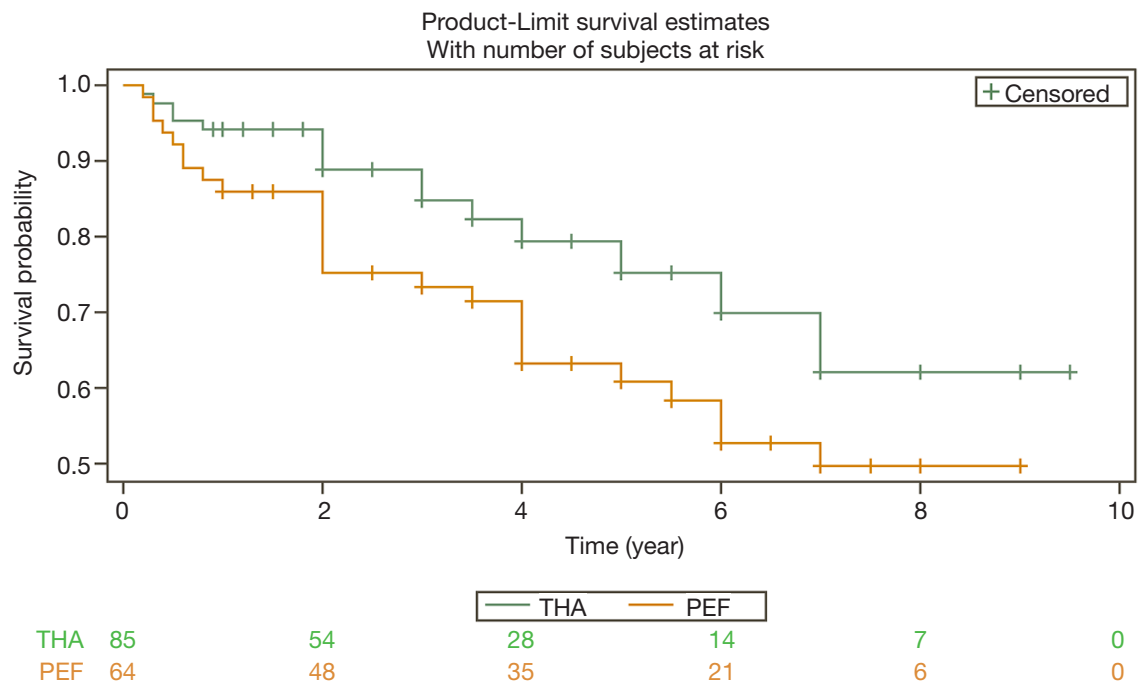

Figure 1 Overall survival of the patients treated with THA and PEF according to the Kaplan-Meier analysis. Data at the bottom represent the number of surviving patients at each time point (Censored data =6). THA, total hip arthroplasty; PEF, percutaneous external fixation.

Table 5 Association of prognostic factors and overall survival

\begin{tabular}{|c|c|c|c|c|c|}
\hline Variates & $B$ value & SE & $\mathrm{P}$ & $\mathrm{HR}$ & $95 \% \mathrm{Cl}$ \\
\hline Age at admission & 0.089 & 0.032 & 0.005 & 1.094 & $1.027-1.166$ \\
\hline ASA score & 0.348 & 0.302 & 0.249 & 1.417 & $0.783-2.565$ \\
\hline Anesthesia type & 0.479 & 0.381 & 0.172 & 1.819 & $0.922-2.174$ \\
\hline \multicolumn{6}{|l|}{ Fracture classification } \\
\hline I & 10.534 & 1384.000 & 0.993 & 0.000 & $0.000-0.000$ \\
\hline II & 0.510 & 0.482 & 0.290 & 1.666 & $0.647-4.289$ \\
\hline III & 0.717 & 0.487 & 0.140 & 2.050 & $0.789-5.329$ \\
\hline IV & 1.206 & 0.489 & 0.013 & 3.342 & $1.280-8.726$ \\
\hline Surgical mode & -1.114 & 0.509 & 0.028 & 0.328 & $0.121-0.890$ \\
\hline Cardio-vascular disorder & 0.194 & 0.423 & 0.645 & 1.215 & $0.530-2.783$ \\
\hline Respiratory disorder & 0.339 & 0.581 & 0.092 & 1.661 & $0.670-4.226$ \\
\hline Metabolic disorder & 0.588 & 0.344 & 0.087 & 1.800 & $0.917-3.533$ \\
\hline Neurological disorder & -0.022 & 0.854 & 0.979 & 0.978 & $0.183-5.219$ \\
\hline
\end{tabular}

SE, standard error; HR, hazard ratio; Cl, confidence interval; ASA, American Society of Anesthesiologists; TFIS, time from injury to surgery. 
is one of the most thorough studies on the subject, with abundant covariates related to the clinical outcome.

Among all of the covariates, the surgical mode was identified to be a pivotal prognostic factor influencing both hip function recovery and overall survival, and the D-dimer level was found to be a crucial prognostic factor influencing 1 -year mortality and overall survival. In addition, the leukocyte count was a factor affecting 1-year mortality. Overall survival was also influenced by age at admission and fracture classification, suggesting that the general preoperative functional indicators age and fracture type, not comorbidities, were independent predictors of midterm survival. Although the incidence of comorbidities in elderly IHF patients (pulmonary, cardiac and neurological diseases, hypertension, diabetes, etc.) can reach $80-95 \%(3,4,11,38)$, $(40,41)$, some studies have reported that, regardless of the surgical treatment selected, pneumonia and heart failure cause significantly high rates of mortality $(14,35,42)$. The results of our study suggest that four types of common comorbidities have a negligible prognostic influence on the survival rate, 1-year all-cause mortality rate, and hip function recovery at the last follow-up. This unexpected finding is not consistent with previous results (43-45).

Our study also presents valuable evidence on the timing of surgical intervention in elderly IHF patients. It is well believed that surgery should be performed as soon as the patient's medical condition allows if appropriate staff and facilities are available $(46,47)$. Some studies have suggested that TFIS is closely related to the occurrence of complications and the clinical outcome $(34,35,41,48)$. In this study, we further analyzed the impact of TFIS on overall survival in a Cox regression model, and the results showed no significant prognostic influence $(\mathrm{P}=0.69)$. Similarly, no significant influence on the 1-year mortality rate was found using a logistic regression model $(\mathrm{P}=0.59)$. These results suggest that TFIS is not a dominant factor in the survival rate, as some other studies have concluded $(42,49)$.

The strengths of the study are that this standard retrospective cohort study was carefully designed and performed. In addition, the mean follow-up period of 5.1 years could be considered to represent mid- to longterm clinical outcomes in elderly patients over 80 years old. In addition, the covariates of the large number of consecutive patients were comprehensively recorded and analyzed using different regression models, ensuring the validity and robustness of the results of this study. There are two limitations in this study. The first is the intraclass heterogeneity resulting from classifying the preoperative comorbidities into four main disorders, which might increase the bias. The second is that the patients in the PEF cohort demonstrated worse physical condition than those in the THA cohort preoperatively, and the difference in ASA score had a definitive influence on the decision-making of anesthesia type and surgical mode. This heterogeneity seemed to be related to the general clinical outcome, as discovered in this work. However, a multivariate Cox proportional hazards model was employed to adjust these preoperative differences, and specific prognostic factors were identified.

\section{Conclusions}

Despite the nonsignificant difference in 1-year all-cause mortality, THA demonstrated superior midterm survival and hip function recovery in elderly IHF patients compared with PEF. Predictive factors, including age at admission, fracture classification, D-dimer level and surgical mode, are associated with the overall survival of IHF in high-risk, elderly patients.

\section{Acknowledgments}

We thank Ms. Changfeng Yang, Yuxia Yuan, Yunyun Kuang, Dekun Yi, and Mr. Linyu Lai from the Department of Orthopedics, Xinqiao Hospital, Army Medical University, for assistance in data recording and clinical follow-up. Professional editing service was performed by American Journal Experts.

Funding: This study was supported by grants from the Technological Innovation and Application Demonstration Project of Chongqing (cstc2018jscx-msyb0541), Innovative Technology in Military and Clinical Medicine (2018JSLC0035), Central Committee Guiding Local Technology Development Project (0028), and Continual Medical Education Project of Chongqing (2020-04-07-067).

\section{Footnote}

Reporting Checklist: The authors have completed the STROBE reporting checklist. Available at http://dx.doi. org/10.21037/atm-20-8037

Data Sharing Statement: Available at http://dx.doi. org/10.21037/atm-20-8037

Conflicts of Interest: All authors have completed the ICMJE 
uniform disclosure form (available at http://dx.doi. org/10.21037/atm-20-8037). The authors have no conflicts of interest to declare.

Ethical Statement: The authors are accountable for all aspects of the work in ensuring that questions related to the accuracy or integrity of any part of the work are appropriately investigated and resolved. The study was conducted in accordance with the Declaration of Helsinki (as revised in 2013). The study was approved by the institutional committee of our centers (No. 2019027), and individual consent for this retrospective analysis was waived.

Open Access Statement: This is an Open Access article distributed in accordance with the Creative Commons AttributionNonCommercial-NoDerivs 4.0 International License (CC BY-NC-ND 4.0), which permits the non-commercial replication and distribution of the article with the strict proviso that no changes or edits are made and the original work is properly cited (including links to both the formal publication through the relevant DOI and the license). See: https://creativecommons.org/licenses/by-nc-nd/4.0/.

\section{References}

1. Berry SD, Kiel DP, Colón-Emeric C. Hip Fractures in Older Adults in 2019. JAMA 2019;321:2231-2.

2. Papadimitriou N, Tsilidis KK, Orfanos P, et al. Burden of hip fracture using disability-adjusted life-years: a pooled analysis of prospective cohorts in the CHANCES consortium. Lancet Public Health 2017;2:e239-e246.

3. Zhang C, Feng J, Wang S, et al. Incidence of and trends in hip fracture among adults in urban China: A nationwide retrospective cohort study. PLoS Med 2020;17:e1003180.

4. Brauer CA, Coca-Perraillon M, Cutler DM, et al. Incidence and mortality of hip fractures in the United States. JAMA 2009;302:1573-9.

5. Dodd AC, Sethi MK. Editorial on "Comprehensive geriatric care for patients with hip fractures: a prospective, randomized, controlled trial" published in The Lancet on April 25th, 2015. Ann Transl Med 2015;3:216.

6. Lu Y, Uppal HS. Hip Fractures: Relevant Anatomy, Classification, and Biomechanics of Fracture and Fixation. Geriatr Orthop Surg Rehabil 2019;10:2151459319859139.

7. Fox KM, Magaziner J, Hebel JR, et al. Intertrochanteric versus femoral neck hip fractures: differential characteristics, treatment, and sequelae. J Gerontol A Biol Sci Med Sci 1999;54:M635-M640.
8. Healy WL, Iorio R. Total hip arthroplasty: optimal treatment for displaced femoral neck fractures in elderly patients. Clin Orthop Relat Res 2004;(429):43-8.

9. Johansson T. Internal fixation compared with total hip replacement for displaced femoral neck fractures: a minimum fifteen-year follow-up study of a previously reported randomized trial. J Bone Joint Surg Am 2014;96:e46.

10. Duriez P, Devaux T, Chantelot C, et al. Is arthroplasty preferable to internal fixation for the treatment of extracapsular fracture of the upper femur in the elderly? Orthop Traumatol Surg Res 2016;102:689-94.

11. Berry SD, Rothbaum RR, Kiel DP, et al. Association of Clinical Outcomes with Surgical Repair of Hip Fracture vs Nonsurgical Management in Nursing Home Residents With Advanced Dementia. JAMA Intern Med 2018;178:774-80.

12. Gjertsen JE. Should Total Hip Arthroplasty Be Used for Hip Fracture? N Engl J Med 2019;381:2261-62.

13. Hassankhani EG, Omidi-Kashani F, Hajitaghi H, et al. How to Treat the Complex Unstable Intertrochanteric Fractures in Elderly Patients? DHS or Arthroplasty. Arch Bone Jt Surg 2014;2:174-9.

14. Mavrogenis AF, Panagopoulos GN, Megaloikonomos PD, et al. Complications After Hip Nailing for Fractures. Orthopedics 2016;39:e108-e116.

15. Smith A, Denehy K, Ong KL, et al. Total hip arthroplasty following failed intertrochanteric hip fracture fixation treated with a cephalomedullary nail. Bone Joint J 2019;101-B:91-6.

16. Zeng X, Zhan K, Zhang L, et al. Conversion to total hip arthroplasty after failed proximal femoral nail antirotations or dynamic hip screw fixations for stable intertrochanteric femur fractures: a retrospective study with a minimum follow-up of 3 years. BMC Musculoskelet Disord 2017;18:38.

17. Cowan R, Lim JH, Ong T, et al. The Challenges of Anaesthesia and Pain Relief in Hip Fracture Care. Drugs Aging 2017;34:1-11.

18. Sidhu AS, Singh AP, Singh AP, et al. Total hip replacement as primary treatment of unstable intertrochanteric fractures in elderly patients. Int Orthop 2010;34:789-92.

19. Tucker NJ, Kamath AF. Comparing total hip arthroplasty and hemiarthroplasty in the treatment of hip fracture. Ann Transl Med 2019;7:S259.

20. Saul D, Riekenberg J, Ammon JC, et al. Hip Fractures: Therapy, Timing, and Complication Spectrum. Orthop Surg 2019;11:994-1002.

21. Archibeck MJ, Carothers JT, Tripuraneni KR, et al. Total hip arthroplasty after failed internal fixation of proximal 
femoral fractures. J Arthroplasty 2013;28:168-71.

22. Yuan BJ, Abdel MP, Cross WW, et al. Hip Arthroplasty After Surgical Treatment of Intertrochanteric Hip Fractures. J Arthroplasty 2017;32:3438-44.

23. Enocson A, Mattisson L, Ottosson C, et al. Hip arthroplasty after failed fixation of trochanteric and subtrochanteric fractures. Acta Orthop 2012;83:493-8.

24. Vossinakis IC, Badras LS. Management of pertrochanteric fractures in high-risk patients with an external fixation. Int Orthop 2001;25:219-22.

25. Petsatodis G, Maliogas G, Karikis J, et al. External fixation for stable and unstable intertrochanteric fractures in patients older than 75 years of age: a prospective comparative study. J Orthop Trauma 2011;25:218-23.

26. Moroni A, Faldini C, Pegreffi F, et al. Dynamic hip screw compared with external fixation for treatment of osteoporotic pertrochanteric fractures. A prospective, randomized study. J Bone Joint Surg Am 2005;87:753-9.

27. Kazemian GH, Emami M, Manafi A, et al. External Fixation vs. Skeletal Traction for Treatment of Intertrochanteric Fractures in the Elderly. Trauma Mon 2016;21:e15477.

28. Kazemian GH, Manafi AR, Najafi F, et al. Treatment of intertrochanteric fractures in elderly high risk patients: $d y-$ namic hip screw vs. external fixation. Injury 2014;45:56872 .

29. Andersen E, Jørgensen LG, Hededam LT. Evans' classification of trochanteric fractures: an assessment of the interobserver and intraobserver reliability. Injury 1990;21:3778.

30. Inoue $T$, Misu S, Tanaka $T$, et al. Acute phase nutritional screening tool associated with functional outcomes of hip fracture patients: A longitudinal study to compare MNA-SF, MUST, NRS-2002 and GNRI. Clin Nutr 2019;38:220-6.

31. Luksameearunothai K, Sa-Ngasoongsong P, Kulachote $\mathrm{N}$, et al. Usefulness of clinical predictors for preoperative screening of deep vein thrombosis in hip fractures. BMC Musculoskelet Disord 2017;18:208.

32. Stotts NA, Deosaransingh K, Roll FJ, et al. Underutilization of pressure ulcer risk assessment in hip fracture patients. Adv Wound Care 1998;11:32-8.

33. Doshi HK, Ramason R, Azellarasi J, et al. Functional improvement of self-care in the elderly after hip fracture: is age a factor? Arch Orthop Trauma Surg 2014;134:489-93.

34. Liu Z, Zhang J, He K, et al. Optimized clinical practice for superaged patients with hip fracture: significance of damage control and enhanced recovery program. Burns Trauma 2019;7:21.

35. Riemen AH, Hutchison JD. The multidisciplinary management of hip fractures in older patients. Orthop Trauma 2016;30:117-22.

36. Socci AR, Casemyr NE, Leslie MP, et al. Implant options for the treatment of intertrochanteric fractures of the hip: rationale, evidence, and recommendations. Bone Joint J 2017;99-B:128-33.

37. Chen P, Hung WW. Geriatric orthopedic co-management of older adults with hip fracture: an emerging standard. Ann Transl Med 2015;3:224.

38. Polat M, Arslan A, Utkan A. External fixation (EF) versus hemiarthroplasty (HA) in unstable intertrochanteric hip fractures of elderly. Acta Orthop Belg 2017:83:351-9.

39. Vossinakis IC, Badras LS. The external fixator compared with the sliding hip screw for pertrochanteric fractures of the femur. J Bone Joint Surg Br 2002;84:23-9.

40. Haentjens P, Casteleyn PP. Treatment of unstable intertrochanteric and subtrochanteric fractures in elderly patients. J Bone Joint Surg Am 1989;71:1214-25.

41. Wei J, Zeng L, Li S, et al. Relationship between comorbidities and treatment decision-making in elderly hip fracture patients. Aging Clin Exp Res 2019;31:1735-41.

42. Roche JJ, Wenn RT, Sahota O, et al. Effect of comorbidities and postoperative complications on mortality after hip fracture in elderly people: prospective observational cohort study. BMJ 2005;331:1374.

43. Ju JB, Zhang PX, Jiang BG. Risk Factors for Functional Outcomes of the Elderly with Intertrochanteric Fracture: A Retrospective Cohort Study. Orthop Surg 2019;11:643-52.

44. Niessen R, Bihin B, Gourdin M, et al. Prediction of postoperative mortality in elderly patient with hip fractures: a single-centre, retrospective cohort study. BMC Anesthesiol 2018;18:183.

45. Sheehan KJ, Sobolev B, Guy P. Mortality by Timing of Hip Fracture Surgery: Factors and Relationships at Play. J Bone Joint Surg Am 2017;99:e106.

46. Lee DJ, Elfar JC. Timing of hip fracture surgery in the elderly. Geriatr Orthop Surg Rehabil 2014;5:138-40.

47. Orosz GM, Magaziner J, Hannan EL, et al. Association of timing of surgery for hip fracture and patient outcomes. JAMA 2004:291:1738-43.

48. Fu MC, Boddapati V, Gausden EB, et al. Surgery for a fracture of the hip within 24 hours of admission is independently associated with reduced short-term post-operative complications. Bone Joint J 2017;99-B:1216-22. 
49. Mitchell SM, Chung AS, Walker JB, et al. Delay in Hip Fracture Surgery Prolongs Postoperative Hospital Length of Stay but Does Not Adversely Affect Outcomes at 30

Cite this article as: Zhang J, Zhu J, Liu Z, Zhang Y, Jin Y, Wang M, Zhang X, He K, Zhang Y. Predictive factors associated with the clinical outcome of intertrochanteric hip fracture in high-risk elderly patients treated with total hip arthroplasty versus percutaneous external fixation. Ann Transl Med 2021;9(9):740. doi: 10.21037/atm-20-8037
Days. J Orthop Trauma 2018;32:629-33. 